\title{
Asbestos exposure, asbestosis, and asbestos-attributable lung cancer
}

\author{
Robert N Jones, Janet M Hughes, Hans Weill \\ Departments of Medicine and Biostatistics and Epidemiology, Tulane University, \\ New Orleans, Louisiana, USA
}

Introductory article

\section{Is lung cancer associated with asbestos exposure when there are no small opacities on the chest radiograph?}

\author{
P Wilkinson, DM Hansell, J Janssens, M Rubens, RM Rudd, A Newman Taylor, J C McDonald
}

The study was designed to test the hypothesis that the risk of lung cancer from asbestos exposure is confined to persons with radiographic evidence of pulmonary fibrosis. Occupational and smoking histories were obtained from 271 patients with a confirmed diagnosis of primary lung cancer and 678 referents (279 with other respiratory disease and 399 with cardiac disease). Histories were reviewed blind to assess the timing, duration, and probability of exposure to asbestos. To allow for a lag between asbestos exposure and the development of lung cancer, subjects were classified by the time they had spent in an occupation entailing definite or probable exposure more than 15 years before diagnosis. The presence and extent of fibrosis was assessed blindly from chest radiographs by three readers and scored for small opacities with the ILO 1980 International Classification of Radiographs of the Pneumoconioses. 93 (34.3\%) cases had worked in an occupation with definite or probable asbestos exposure compared with 176 (25.8\%) referents (crude odds ratio for lung cancer 1.49, 95\% Cl 1.09-2.04). After adjustment for age, sex, smoking history, and area of referral, the odds ratio (95\% Cl) was 2.03 $(1.00-4.13)$ in the subgroup of 211 with a median ILO score for small parenchymal opacities of 110 or more, and $1.56(1.02-2.39)$ in the 738 with a score of 0/1 or less (ie, those without radiological evidence of pulmonary fibrosis). These results suggest that asbestos is associated with lung cancer even in the absence of radiologically apparent pulmonary fibrosis. (Lancet 1995;345:1074-8)

\begin{abstract}
Asbestos is the most studied of all occupational carcinogens and, apart from tobacco, the most studied cause of lung cancer. It may therefore surprise the general reader that there is an important area of uncertainty about the relationship between inhaled asbestos and the resulting increase in risk of lung cancer. At issue is whether asbestos-attributable lung cancers are always associated with asbestos-induced lung fibrosis - that is, asbestosis. This uncertainty has engendered a heated controversy, fuelled by important implications for regulation, workers' compensation, and litigation. A review of the evolution of knowledge about exposure to asbestos and increased lung cancer risk may be helpful in understanding both the sources of the uncertainty and the significance of the introductory article.
\end{abstract}

\section{Epidemiology}

The earliest reports - and for many years the only reports - presented cases of coexisting asbestosis and lung cancer. ${ }^{1}$ By their very nature such case reports, no matter how numerous, cannot establish a causal relationship, but they were, in fact, reports of cancer associated with the disease asbestosis - not just with asbestos exposure. The first systematic inquiry was published in 1949 in Merewether's Annual Report of the Chief Inspector of Factories for the Year $1947 .^{2}$ In a series of postmortem lung specimens from patients with asbestosis and silicosis he observed that the former had a substantially higher rate of lung cancer than the latter, $13.2 \%$ versus $1 \cdot 3 \%$. As in the earlier case reports, the observed association was between asbestosis and lung cancer. According to the 1965 account of Merewether's successor, Buchanan, "At that time, an attitude of caution was adopted and no formal claims of any association were made". ${ }^{3}$

The first study of lung cancer risks in an occupational cohort (manufacturing workers in an insulation products plant in Rochdale) was published by Doll in $1955 .{ }^{4}$ All 11 of the lung cancers occurred in workers with asbestosis. The early evidence on causation was thus in the context of the disease asbestosis.

Doll's report greatly strengthened the case for a causal relationship and sparked intense interest in identifying other populations at risk. While the earlier observations 
were in subjects involved in the manufacture of asbestos products, subsequent work soon revealed excess lung cancer rates in the users of those products, especially in the construction and shipbuilding industries. The typical contribution was a retrospective mortality study of workers in a craft (for example, insulation workers) or a setting (for example, dockyards). There were no data on the intensity of exposures so length of work was used as a surrogate for lifetime exposure, sometimes with an additional factor based on ranking the perceived dustiness of various trades. Smoking histories and other elements of personal risk or health status were almost never available. It was easy to assume that, because the cohorts were defined only in terms of exposure, any increase in mortality from lung cancer was a direct result of exposure per se. When smoking data were incorporated, it was assumed that the observed relationships were between smoking and exposure.

Selikoff's cohort study of US insulation workers provided the most widely quoted estimates of lung cancer mortality from asbestos "exposures" and cigarette smoking. ${ }^{56}$ For many years, whether in estimates of the proportion of occupational lung cancers, in projections of their future numbers, or in disputed causation in individual cases, experience of the insulation workers was routinely offered as quantifying the carcinogenic effects of "exposure". 7 The interaction between "exposure" and smoking was multiplicative or synergistic, which was regarded as evidence that asbestos was implicated in the majority of lung cancer deaths among the "exposed population". When this was controverted it was usually on the grounds that the insulation workers were the most heavily exposed group, and their cancer experience would not apply in individuals or groups with lesser exposures. Then, in a 1987 publication, Kipen and others (including Selikoff) reported surgical or necropsy results in a series of consecutive cases of insulation workers with lung cancer. ${ }^{8}$ Of 138 cases with acceptable tissue and radiographs, all had histological evidence of lung fibrosis and $113(82 \%)$ also had radiographic evidence of fibrosis. The mortality experience from lung cancer in the insulation workers therefore derived not merely from exposure to asbestos but from asbestosis.

The "synergism" shown in Selikoff's mortality data is still being offered as evidence for an effect of exposure per se, independent of asbestosis. Consider, however, the two models shown in the figure. The interactions with smoking could be either additive or multiplicative. In the light of the study by Kipen et $a l,{ }^{8}$ model 2 is the more accurate representation of the mortality data of the insulation workers. When all lung cancers in the insulation workers occur in lungs affected with asbestosis, then all of the excess lung cancers must also be there.

There were continuing observations of the association of lung cancer with asbestosis. In 1965 Buchanan concluded that "... there is a special risk of an intrathoracic tumor if asbestosis is also present in the lungs", ${ }^{3}$ and in 1969 Newhouse and Wagner reported moderate or severe histological asbestosis in necropsy specimens of all the confirmed lung cancer cases from ex-workers in an asbestos factory. ${ }^{9}$

Other investigators began to incorporate estimates of individual cumulative exposure in cohort studies. As is the case with cigarette smoke, lung cancer risks were found to be related to cumulative asbestos exposure. In populations with morbidity data, excess deaths from lung cancer generally started at about the same cumul-
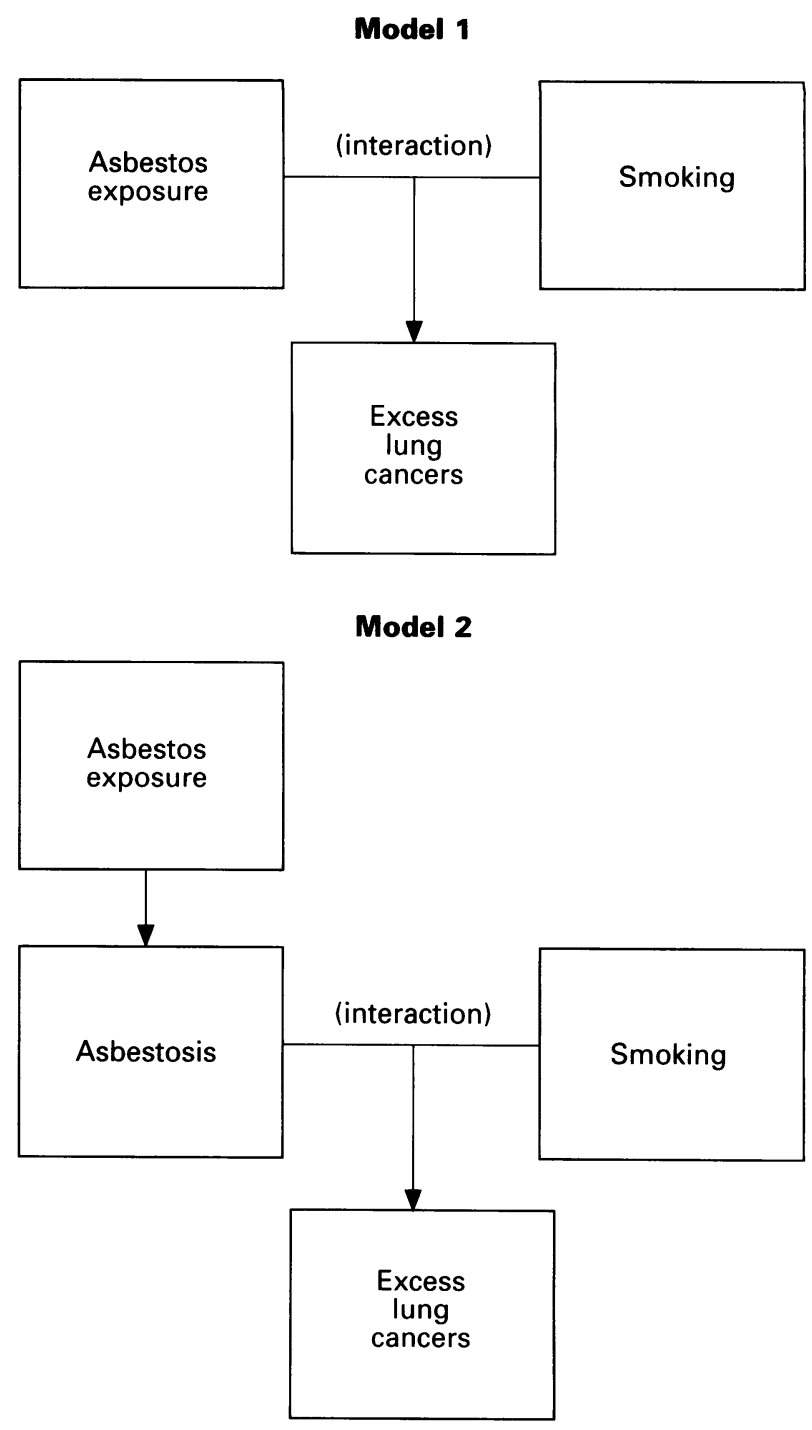

Competing models relating exposure, smoking, and cancers. Model 1 is drawn from a data set that lacks information on which exposed subjects have asbestosis. When it is known that the excess cancers occur in subjects who also have asbestosis, model 2 provides a better depiction.

ative exposure levels as those at which asbestosis began to be detected. ${ }^{10}$

In 1988 Hughes and Weill reported results of a prospective study in which there were both individual estimates of cumulative dust exposure and health data, including smoking and radiographic status at the outset of observation. ${ }^{112}$ The subjects were 839 men in two asbestos cement plants who had been examined in a cross sectional morbidity study in 1969 . Twenty or more years after hire no excess of lung cancer was found among workers without radiographic evidence of lung fibrosis, nor was there a trend in risk by level of cumulative exposure to asbestos among such workers. By contrast, employees with small opacities ( $\geqslant 1 / 0$; ILO classification) experienced a significantly increased risk of lung cancer (nine observed deaths compared with $2 \cdot 1$ expected) even though their exposures to asbestos were similar to the exposures of long term workers without opacities. The authors concluded that the excess risk of lung cancer in that population was restricted to workers with radiographic evidence of asbestosis, a finding consistent with the view that asbestos is a lung carcinogen because of its fibrogenicity.

In 1989 Sluis-Cremer and Bezuidenhout reported a necropsy series of 339 South African amphibole asbestos miners. ${ }^{13}$ Heavy smoking, age, and the presence of 
histological asbestosis were all significantly associated with the presence of bronchial cancer. Of the 35 cases of bronchial cancer, 24 had asbestosis. In the 11 cases of bronchial cancer which occurred in men without asbestosis, all were smokers. Standardised proportional mortality rates indicated no excess of bronchial cancer in 302 exposed men without asbestosis, although these rates were progressively raised in men with increasing severity of asbestosis. Of the four exposure variables introduced separately into a logistic regression model, "years of exposure" made a small but significant contribution, "residence time" marginally failed to achieve a 5\% level of significance, and neither cumulative fibre exposure (fibre years) nor "residence time weighted dosage" contributed significantly. The authors concluded that, in the absence of asbestosis detected at necropsy, a bronchial cancer in a man exposed to asbestos is unlikely to be due to exposure to asbestos.

\section{Introductory article}

Wilkinson and colleagues ${ }^{14}$ state the research question in the title of their paper. The reported work is a casecontrol study, and case-control studies are known to have some inherent limitations in establishing cause and effect, but these are beyond the scope of this review. Patients admitted to hospital with lung cancer were the cases and hospital patients with other lung diseases or heart conditions were the controls. Exposures to asbestos were determined by interviewing subjects and categorising their jobs according to a listing that had proved to be useful in a North American study of mesothelioma. Radiographic small opacities were determined using the ILO 1980 classification. ${ }^{15}$ To prevent the readers from identifying cases and controls, up to two quadrants on the radiographs of the cancer patients were masked to obscure the tumour, and the radiographs of the controls had to be similarly masked.

Setting aside the bias in selecting subjects only after they have become ill, this design entailed potential problems in two areas. Even the fact of exposure and, much more, its severity is difficult to determine retrospectively by interviewing patients. Assessment of exposure is far more accurate when exposure, rather than illness, is the point of departure. Secondly, a comparison of diffuse lung abnormality of patients with lung cancer with that of patients with heart or other lung disease all selected because of hospital admission not asbestos exposure - introduces so many extraneous causes that diffuse abnormality must inevitably become a poor marker of lung fibrosis and a questionable indicator of asbestos effects.

The authors actually ignored their subjects' assertions concerning whether or not each was exposed to asbestos. Why it was preferable to use a mesothelioma study job classification is not explained. The dose-response relationships of asbestosis and asbestos-attributable lung cancer are similar to each other but differ greatly from those of mesothelioma. Only the jobs in the authors' "definite" category - asbestos production, heating trades, and insulation work - inspire much confidence that each individual so classified would probably have had moderate or heavy exposure. The controls proved to have greater prevalence of "definite" exposure than the cases! Jobs listed in the "probable" and "possible" categories included many for which there is inadequate evidence of asbestos-related lung cancer. In the analyses the authors combined "definite" and "probable" as both a categorical variable and a criterion for computing length of exposure.
Potential problems in the radiographic assessment in the study included possible misclassification of subjects with respect to small opacities and doubts about the very nature of small opacities in this study population. Obscuring as much as half of the lungs (two quadrants) has an undetermined but potentially large effect on the ability to classify radiographs properly. ILO readings require more than just the ability to see and grade small opacities. At the outset the reader must decide whether small opacities probably have some other cause, in which case they are not classified and the radiograph will be categorised as negative. Masking the radiographs would be expected to reduce the chance of a correct judgement, leading to both false positive and false negative assessments. In fact, Wilkinson et al failed to find a relation between exposure to asbestos and the presence of small opacities. For example, although 55 women were judged to have small opacities, only eight were considered to have been exposed to asbestos. This may reflect imprecisions in the assessment of exposure, radiographic status, or both. It might be argued that only the negative radiographs mattered in defining lack of fibrosis, and that false positive judgements could not produce spurious elevations of odds ratios in the radiographically negative subjects. For this to be correct there should be no systematic bias of radiographic status according to exposure status. The argument would at least convert the observed lack of exposure effects on the radiographs from a liability to an asset. The potentially misleading effect of false negatives remains, however, and at some point one must become uncomfortable with the complete divorce of radiographic abnormality and asbestos effect. Why not study asbestos and cancer risks in subjects whose radiographic status is relatable to asbestos exposure?

Wilkinson et al reported several observations that met tests of statistical significance:

- A crude odds ratio ( $95 \% \mathrm{CI}$ ) for lung cancer of 1.49 (1.09 to 2.04 ). This was obtained by combining the definite and probable exposure categories (the definite category alone would have produced an odds ratio of $<1 \cdot 0)$.

- An odds ratio, adjusted for age, sex, and smoking, of $1 \cdot 66(1 \cdot 15$ to $2 \cdot 38)$. This was obtained using patients with definite or probable exposure more than 15 years before diagnosis, then dichotomising on "a simple classification of exposure - any relevant job against none," not further explained.

- Adjusted odds ratios of 2.03 for those with small opacities of profusion $1 / 0$ or greater, and 1.56 with $0 / 1$ or $0 / 0$ - but the latter just attained significance $(1.02$ to 2.39$)$ and the former just missed it ( 1.00 to $4 \cdot 13)$.

When the adjusted odds ratios (ORs) were further broken down for small opacities of category $0 / 1$ or less and category $0 / 0$ alone, the authors found "evidence that ORs increased with duration of exposure" in both categories. However, in both categories the ORs for less than 10 years' exposure were much higher than the ORs for 10 or more years' exposure, and the ORs of the latter were not significantly $>1.0$ in all subjects or in men aged 40 years or older. This is surely evidence against, not for, a dose-response relationship in groups with "negative" radiographs. In the end, the cited observations rest only on dichotomised exposures, estimated according to a scheme that may be valid for mesothelioma but cannot be presumed so for lung cancer. Under the weight of additional concerns about 
the study of hospital patients with masked radiographs, this work does not provide convincing support for an asbestos-attributable risk in the absence of small opacities.

\section{Broader field of controversy}

Evidence from other lines of enquiry has a bearing on the issue. Reviews and opinions have been and continue to be published, ${ }^{16-23}$ and various arguments are advanced. In the following sections "linkage" refers to the view that asbestos-attributable lung cancers are those that are associated with asbestosis.

\section{LUNG FIBROSIS OF OTHER CAUSES CARRIES AN INCREASED} RISK OF LUNG CANCER

The Brompton Hospital has long been a centre of research on cryptogenic fibrosing alveolitis (CFA), called idiopathic pulmonary fibrosis (IPF) in the USA. Turner-Warwick and her colleagues followed patients with this condition and reported on their causes of death in a 1980 publication. ${ }^{24}$ Lung cancer was found in 20 (9.8\%) of 205 patients with CFA or $12.9 \%$ of the 155 patients in this series followed to death. An excess relative risk for lung cancer of $14 \cdot 1$ was found in patients with CFA compared with the general population of comparable age and sex, allowing for the lengths of follow up of the patients with CFA. The relative risk for male smokers was (observed (O)/expected (E)) 15/ $1 \cdot 06=14 \cdot 2$, and for female smokers $(\mathrm{O} / \mathrm{E}) 2 / 0 \cdot 3=6 \cdot 7$. Only one male and one female non-smoker had lung cancer. These data suggested to the authors that there is an excess risk of lung cancer in patients with CFA that is not wholly accounted for by age, sex, or smoking habit. The distribution of histological types was not obviously different from that found in cases of lung cancer without pulmonary fibrosis.

Nagai and colleagues ${ }^{25}$ reported lung cancer in 31 of $99(31.3 \%)$ patients with IPF. Cancers occurred in $10.7 \%$ of the non-smokers and $38 \%$ of the smokers. Most of the tumours were peripheral in location, frequently in the lower lobes, and the distribution of cell types of lung cancer was similar to that encountered in the general population. The authors speculated that lung cancer in IPF "may result from uncontrolled epithelial proliferation secondary to fibrosis and chronic inflammation ...", with smoking as an additional effect.

A review by Henderson et $a l^{23}$ acknowledged the increased cancer risks of some non-occupational fibroses, but then focused on the different risks in subjects with asbestosis and silicosis, going back to the Merewether report cited above. The idea, not explicitly stated, seems to be that if two agents each produce fibrosis but have different carcinogenic potency, this detracts from a carcinogenic role of fibrosis. The argment would have some weight if silica produced a fibrosis that did not increase the risk of lung cancer. However, recent evidence clearly indicates that silicosis does carry an excess risk of lung cancer, ${ }^{2627}$ so excess risks attach to both occupational and non-occupational fibroses.

\section{DO REGISTRY STUDIES SUPPORT NON-LINKAGE OF} ASBESTOSIS AND ASBESTOS-ATTRIBUTABLE LUNG CANCERS? Irvine and colleagues ${ }^{28}$ used the Scottish Cancer Registry and postcodes to identify all men diagnosed with lung cancer. They estimated that $5 \%$ of all lung cancers in the shipbuilding area around Glasgow were asbestos- related. Because of the small number of recorded cases of asbestosis, the authors concluded that this condition is not a prerequisite for cancer. Tumour registry data, like death certificates, will always underestimate the prevalence of asbestosis, so such studies cannot provide persuasive evidence of non-linkage.

Barroetavena et al examined rates of compensation for asbestos-related lung cancer, asbestosis, and mesothelioma in British Columbia and New South Wales. ${ }^{29}$ Starting from the premise that asbestos-attributable lung cancers should number about twice the mesotheliomas, they concluded that there was serious under-registration of the lung cancers and that, with the "large numbers" of cases of asbestosis already registered, there could hardly be enough additional ones to explain the supposed excess of unregistered lung cancers. There is, however, no reason to believe that there is a constant ratio of asbestos-attributable lung cancers to mesotheliomas, one that applies to populations with different exposure intensities and time courses, fibre type mixes, and smoking habits, all of which have differential effects on the incidences of the two neoplasms. This being so, the authors have failed to prove the existence of "unrecognised" asbestos-induced lung cancers. As for the "large numbers" of registered asbestosis cases, they were in fact less numerous than the mesotheliomas. Asbestosis nowadays is rarely disabling or fatal, so neither compensation nor death certificate data can provide adequate estimates of its prevalence.

A recent Australian paper ${ }^{30}$ examined cancer registry data in relation to exposure to crocidolite and smoking, incorporating also the theory that histological type indicates causation. There was no determination of which cases had asbestosis. Finding that squamous cancers were related to cumulative asbestos exposure, the authors concluded that this weakens the case for linkage with asbestosis, arguing that fibrosis-associated cancers are adenocarcinomas and that squamous cancers are not peripheral and are therefore unrelated to fibrosis. However, the cited studies of idiopathic fibrosis ${ }^{2425}$ found that histological type distributions were not very different from the general and, as discussed below, linkage does not require that asbestos-attributable tumours be "scar carcinomas".

\section{FIBRE BURDEN STUDIES IN THE DEMONSTRATION OF} CAUSATION

One older study of this type was recently cited as supporting non-linkage. In 1986 Warnock and Isenberg $^{31}$ reported an attempt to distinguish between lung cancers related and unrelated to asbestos by identifying and quantifying fibres in the lungs of 75 men with lung cancer. All but eight had some history of exposure to asbestos. Cases were grouped according to concentration of combined amosite and crocidolite in fibres/gram dry lung: $<10^{5}(\mathrm{n}=15), 10^{5}-10^{6}(\mathrm{n}=35)$, $>10^{6}(\mathrm{n}=25)$. Age, smoking history, latent period, and type and location of tumours were similar in all three groups. Of 62 cases evaluated for fibrosis, none of 14 in the low concentration group, seven of $29(24 \%)$ in the intermediate group, and five of $19(26 \%)$ in the high group had "macroscopic fibrosis" (wording in the text) or "asbestosis" (wording in the abstract). The authors concluded that subjects in the intermediate and high concentration groups may have been at "increased risk" for cancer, even when they did not have asbestosis. They stated that, because large burdens of asbestos do not always cause asbestosis, "asbestosis may be a poor marker of fiber-related lung cancer". The flaw in this 
reasoning is that all of the subjects had cancer, and Warnock and Isenberg had no evidence of "increased" (or excess) cancer risk attributable to asbestos or any other factor. Their results show only that macroscopic fibrosis, or "asbestosis" as they define it, is associated with larger fibre burdens in patients with lung cancer, a matter not in dispute and not relevant to the issue of linkage. Of more interest, however, is their observation that "diffuse microscopic fibrosis" (which sounds a lot like asbestosis) was present in 50 of 60 cases in the intermediate and high fibre burden groups, suggesting that if there were independent evidence that those cancers were attributable to asbestos, the paper would support linkage, not non-linkage.

In a 1993 Finnish report 108 cases of resected lung cancer were studied. ${ }^{32}$ The objective was to relate location of lung cancers to asbestos exposure and the presence or absence of asbestosis. Job histories were evaluated by industrial hygienists. Transmission electron microsopic counts of asbestos fibre were performed and generally agreed with the hygienists' evaluations. Based on the history, $36 \%$ had definite or probable asbestos exposure; fibre burdens consistent with occupational exposure were found in $31 \%$. Only $4 \%$ of patients had never smoked. Eight cases had histologically confirmed asbestosis and one had diffuse visceral pleural fibrosis. These nine each had either probable or definite occupational exposure to asbestos; seven had cancer in the lower lobes and two in the upper lobes. In the whole group tumour location could be determined in 106 cases: $58 \%$ were in the upper lobes, $3 \%$ in the middle lobes, and $39 \%$ in the lower lobes. Smoking did not influence lobar distribution and asbestos exposure did not influence cell type (the contrary finding was published in 1994 by the same group). Exposure to asbestos was more likely to have occurred in those with lower lobe tumours, even when those with asbestosis or pleural fibrosis were excluded. The inference is that lower lobe tumours are, to a degree, attributable to exposure, even in the absence of asbestosis. However, as with the study of Warnock and Isenberg, there is no independent way of determining that lower lobe tumours in exposed subjects represent excess cancers that would not have developed without exposure. While these investigators found a relationship between tumour location and exposure, others have found the opposite. It is difficult to accept that this proves a causal link between asbestos exposure per se and lung cancer risk. A cautionary note: operability is itself related to site of cancer origin, the right upper lobe having both the highest rate of tumour development and the lowest rate of resectability. How this might have influenced the results is not clear, but selection bias is the greatest potential hazard in surgical or necropsy series.

The same group of investigators published a report in 1994 on asbestos exposure and risk of lung cancer in a general urban population. ${ }^{33}$ One hundred and thirteen surgically treated men with lung cancer were compared with 297 necropsy cases used as controls. Odds ratios were calculated according to lung asbestos fibre burden for all cell types of lung cancer and for lobe of origin. Departing from the standard practice of making cases and controls as comparable as possible, tissue was sampled from the lobe of origin in the cases and from the left upper lobe in the controls on the assumption that fibre burdens would be higher in the upper lobes. Lung cancer risk was found to be related to lung fibre burden. In cases, however, fibre burdens were found to be higher in the lower lobes. The failure to match the controls for sampled lobe thus biased the comparison of fibre burden. Risks associated with fibre concentration were also found to be higher for adenocarcinoma than squamous carcinoma and higher for lower than upper lobe tumours. The presence of asbestosis was determined from the surgical specimen in the cases but was not determined for the controls. Smoking information was far more complete for lung cancer patients than for controls and the smoking adjustment was made more difficult because only two lung cancer patients were non-smokers. When those with asbestosis were removed from the analysis, the increased risk of lung cancer in relation to fibre burden was no longer statistically significant. The findings raise an important question beyond the linkage issue - namely, are squamous cancers and upper/middle lobe cancers not attributable to asbestos on the grounds that their risk estimates were non-significant in this study? The evidence relating asbestos exposure to lobar origin and to cell type is both weak and inconsistent, and the prevailing view is that these features cannot be used to determine attributability. If the negative findings of the Finnish studies are to be discounted in light of contrary findings from other studies, it is difficult to argue against the same approach for the positive findings. A paper published by Baker ${ }^{34}$ in 1991 provides a good perspective on the subject.

ANIMAL STUDIES CONSISTENTLY DEMONSTRATE LINKAGE OF ASBESTOSIS AND EXPERIMENTAL TUMOURS

The great preponderance of evidence indicates that tumour incidence in animals exposed to asbestos dust is increased only in the presence of fibrosis. ${ }^{3536}$ Not all of the tumours are cancers, and there are always reservations about the applicability of animal data to humans, but these results provide support for linkage.

MOST LUNG CANCERS OCCUR IN LARGE CENTRAI

BRONCHI, EVEN IN CASES WITH ASBESTOSIS

This would be a telling point if the concept of linkage required asbestos-attributable carcinomas to be "scar cancers" which are peripheral adenocarcinomas that develop in relation to pre-existing scars. There is, however, no such requirement. An inflammatory response causing cell proliferation that induced both fibrogenesis and carcinogenesis would produce linkage, even with fibrosis and cancer expressed at different sites in the lung. A recent review on basic biological mechanisms of asbestos-related diseases states: "Asbestosis is an inflammatory and fibrotic process of the alveolar structures mediated, at least in part, by cytokines released by activated alveolar macrophages ... cancer and asbestos is more vexing. The processes of inflammation, fibrosis, and carcinogenesis appear to be closely intertwined. For example, proto-oncogenes such as c-sis (PDGF-B-chain) are upregulated in activated alveolar macrophages from fibrotic lungs; these and possibly others may play an important role in asbestos carcinogenesis". ${ }^{37}$

ARE PLEURAL PLAQUES A MARKER FOR ASBESTOS-ATTRIBUTABLE LUNG CANCERS?

Plaques develop after lower exposures to asbestos and are therefore much more prevalent than asbestosis in exposed populations. Harber et $a l^{38}$ found no excess lung cancer risk in asbestos exposed men with plaques compared with similarly exposed men who lacked plaques 
LEARNING POINTS

\section{* Lung fibrosis of many causes - known and unknown - is associated with increased risk of lung cancer.}

* The much discussed synergism between asbestos "exposure" and smoking found in mortality studies of insulation workers turns out to be a synergism involving asbestosis, not just asbestos exposure.

* The site of origin and cell type of a lung cancer are not regarded as reliable indicators
of causation (or non-causation) by asbestos.

* In asbestos inhalation experiments animals develop excess lung tumours only when lung fibrosis is also produced.

* Pleural plaques have not proved to be a reliable marker for increased risk of lung cancer.

* Excess lung cancer deaths in populations exposed to asbestos are generally first detected at about the same cumulative exposure levels as those at which asbestosis begins to appear.

(matching was also on race and smoking, and men with plaques plus asbestosis were excluded). In the asbestos cement workers studied by Hughes and Weill ${ }^{12}$ there was no significantly increased risk in those with pleural disease only.

A second approach is to examine the risk of lung cancer in persons with plaques in comparison with the risk in unexposed persons or the general population. An increased risk of lung cancer associated with plaques (after exclusion of cases who also have overt asbestosis) would constitute evidence against linkage with asbestosis. In a 1993 review $^{39}$ Weiss examined six cohort studies, four case-control studies, and three necropsy studies of plaques and lung cancer and found that "the weight of the evidence favors the conclusion that persons with asbestos-related pleural plaques do not have an increased risk of lung cancer in the absence of parenchymal asbestosis".

A prospective study of 1596 Swedish men with plaques was published by Hillerdal in $1994 .^{40}$ Most cases were detected in a longitudinal survey; 166 initially denied asbestos exposure but 86 later confirmed it after they "did some private research". The incidence of lung cancer was compared with the expected incidence from the national cancer registry. An adjustment for smoking habits was based on "national statistics". The adjusted risk ratio for lung cancer for those with plaques but without radiographic asbestosis was $1 \cdot 4(\mathrm{O} / \mathrm{E}=41 / 28 \cdot 2$, $95 \%$ CI 1.04 to 1.97 ). Nine of the 50 observed lung cancers were in men with radiographic asbestosis. Weaknesses of this study include the fact that $15 \%$ of cases with plaques were discovered through sources other than the community survey (potential clinical bias), use of miniature radiographs (probable underdetection of asbestosis), and possibility of imprecise smoking adjustment. An editorial published in the same journal issue reviewed the (generally contrary) findings of other radiographic studies and necropsy studies and concluded: "This study is just another study that has failed to demonstrate convincing evidence of a significant risk of lung cancer associated with pleural plaques without asbestosis".41

Conclusions

While the issue of whether asbestosis is a necessary precursor to asbestos-attributable lung cancer cannot at this time be considered settled, the weight of the available evidence strongly supports this proposition. The best evidence comes from studies of well defined exposed populations, using a prospective cohort design, in which there are available estimates of past exposure to asbestos and smoking histories, with valid radiographic (or histopathological) assessment for diffuse lung fibrosis and adequate mortality follow up data. Case-control and fibre burden studies have thus far been methodologically weak, with selection bias likely for both types of investigations. The absence of standardised methodology for tissue fibre counting has been an especially serious problem, particularly in light of the pathogenetic importance of differing fibre dimensions and fibre persistence in the lung.

The linkage between inflammation/fibrosis and carcinogenesis is plausible in light of recent work on these fundamental biological processes. There is also ample evidence that diffuse lung fibrosis of other causes, known and unknown, is associated with an excess risk of lung cancer. With the present knowledge regarding this probable linkage, for compensation and other decisions in cases where asbestos attributability of lung cancer is the issue, it is reasonable for judgements of attributability to rest on the presence of asbestosis, diagnosed by either radiography or histopathology.

1 Enterline PE. Changing attitudes and opinions regarding asbestos and cancer 1934-1965. Am f Ind Med 1991;20:685-700.

2 Merewether ERA. Asbestosis and carcinoma of the lung. In: Annual Report of the Chief Inspector of Factories for the Year 1947. London: HMSO Stationery Office, 1949.

3 Buchanan WD. Asbestosis and primary intrathoracic neoplasms. Ann NY Acad Sci 1965;132:507-18.

4 Doll R. Mortality from lung cancer in asbestos workers. $\mathrm{Br} \mathcal{F}$ Ind Med 1955;12:81-6.

5 Selikoff IJ, Hammond EC, Seidman H. Mortality experience of insulation workers in the United States and Canada, 1943-1976. Ann NY Acad Sci 1979;330:91-116.

6 Hammond EC, Selikoff IJ, Seidman H. Asbestos exposure, cigarette smoking, and death rates. Ann NY Acad Sci 1979;330:473-91.

7 Asbestos linked cancer rates up less than predicted. 15 April 1992 "News", FNCI 1992;84:560-2.

8 Kipen HM, Lilis R, Suzuki Y, Valciukas JA, Selikoff IJ. Pulmonary fibrosis in asbestos insulation workers with lung cancer: a radiological and histopathological evaluation. $B r$ F Ind Med 1987;44:96-100.

9 Newhouse ML, Wagner JC. Validation of death certificates in asbestos workers. Br f Ind Med 1969;26:302-7.

10 Browne $\mathrm{K}$. A threshold for asbestos related lung cancer. $\mathrm{Br} \mathcal{F}$ Ind Med 1986;43:556-8.

11 Hughes JM, Weill H. Pulmonary fibrosis as a determinant of asbestosinduced lung cancer in a population of asbestos cement workers. In: Proceedings of the VIIth International Pneumoconioses Conference, 
Pittsburgh, 1988. US Department of Health and Human Services, 1990:370-4.

12 Hughes JM, Weill $\mathrm{H}$. Asbestosis as a precursor of asbestos related lung cancer: results of a prospective mortality study. $\mathrm{Br} \mathcal{F}$ Ind Med 1991; 48:229-33.

13 Sluis-Cremer G, Bezuidenhout BN. Relation between asbestosis and bronchial cancer in amphibole asbestos miners. $\mathrm{Br} \mathcal{F}$ Ind Med 1989; 46:537-40.

14 Wilkinson P, Hansell DM, Janssens J, Rubens M, Rudd RM, Taylor AN, McDonald JC. Is lung cancer associated with asbestos exposure when there are no small opacities on the chest radiograph? Lancet 1995;345:1074-8.

15 International Labour Office. Guidelines for the use of ILO international classification of radiographs of pneumoconioses. Occupational Safety and Health Series No. 22 (Rev). Geneva: ILO, 1980.

16 Sluis-Cremer G. The relationship between asbestosis and bronchial cancer. Chest 1980;78(Suppl):380-1.

17 Churg A. Current issues in the pathologic and mineralogic diagnosis of asbestos-induced disease. Chest 1983;84:275-80.

18 Browne $\mathrm{K}$. Is asbestosis or asbestosis the cause of the increased risk of lung cancer in asbestos workers? Br F Ind Med 1986;43:145-9.

19 Commins BT. Estimations of risk from environmental asbestos in perspective. In: Bignon J, Peto J, Saracci R, eds. Non-occupational exposure to mineral fibres, IARC Scientific Publication No. 90. Lyon: IARC, 1989:476-85.

20 Browne $\mathrm{K}$. Asbestos related malignancy and the Cairns hypothesis. $\mathrm{Br}$ f Ind Med 1991;48:73-6.

21 Jones RN. Asbestos and thoracic neoplasms. Semin Roentgenol 1992;27: 94-101.

22 Churg A. Asbestos, asbestosis, and lung cancer. Mod Pathol 1993;6: 509-11.

23 Henderson DW, Roggli VL, Shilkin KB, Hammar SP, Leigh J. Is asbestosis an obligate precursor for asbestos-induced lung cancer? Fiber burden and the changing balance of evidence: a preliminary discussion document. In: Peters GA, Peters BJ, eds. Sourcebook on asbestos diseases (in press).

24 Turner-Warwick M, Lebowitz M, Burrows B, Johnson A. Cryptogenic fibrosing alveolitis and lung cancer. Thorax 1980;35:496-9.

25 Nagai A, Chiyotani A, Nakadate T, Konno K. Lung cancer in patients with idiopathic pulmonary fibrosis. Tohoku $f$ Exp Med 1992;167: 231-7.

26 Smith AH, Lopipero PA, Barroga VR. Meta-analysis of studies of lung cancer among silicotics. Epidemiology 1995;6:617-24.

27 Weill $\mathrm{H}, \mathrm{McD}$ onald JC. Exposure to crystalline silica and risk of lung cancer: the epidemiological evidence. Thorax 1996;51:97-102.

28 Irvine HdeV, Lamont DW, Hole DJ, Gillis CR. Asbestos and lung cancer in Glasgow and the West of Scotland. BMF 1993;306:1503-6. 29 Barroetavena MC, Teschke K, Bates DV. Unrecognized asbestosinduced disease. Am F Ind Med 1996;29:183-5.

30 de Klerk NH, Musk AW, Eccles JL, Hansen J, Hobbs MST. Exposure to crocidolite and the incidence of different histological types of lung cancer. Occup Environ Med 1996;53:157-9.

31 Warnock ML, Isenberg W. Asbestos burden and the pathology of lung cancer. Chest 1986;89:20-6.

32 Karialainen A, Anttila S, Heikkila L, Kyronen P, Vainio H. Lobe of origin of lung cancer among asbestos-exposed patients with or without diffuse interstitial fibrosis. Scand $f$ Work Envirn Health 1993;19: diffuse

33 Karjalainen A, Anttila S, Vanhala E, Vainio H. Asbestos exposure and the risk of lung cancer in a general urban population. Scand $\mathcal{f}$ Work Environ Health 1994;20:243-50.

34 Baker DB. Limitations in drawing etiologic inferences based on measurement of asbestos fibers in lung tissue. Ann NY Acad Sci 1991;643: 61-70.

35 Wagner JC, Berry G, Skidmore JM, Timbrell V. The effects of the inhalation of asbestos in rats. Br f Cancer 1974;29:252-69.

36 Davis JMG, Beckett ST, Bolton RE, Collings P, Middleton AP. Mass and number of fibres in the pathogenesis of asbestos-related lung disease in rats. Br $\mathcal{f}$ Cancer 1978;37:673-88.

37 Rom WN, Travis WD, Brody AR. Cellular and molecular basis of the asbestos-related diseases. Am Rev Respir Dis 1991;143:408-22.

38 Harber P, Mohsenifar Z, Oren A, Lew M. Pleural plaques and asbestosassociated malignancy. 7 Occup Med 1987;29:641-4.

39 Weiss W. Asbestos-related pleural plaques and lung cancer. Chest 1993; 103:1854-9.

40 Hillerdal G. Pleural plaques and risk for bronchial carcinoma and mesothelioma - a prospective study. Chest 1994;105:145-50.

41 Smith DD. Plaques, cancer and confusion. Chest 1994;105:8-9. 